\title{
Endoscopic photoacoustic microscopy
}

Joon-Mo Yang, Konstantin Maslov, Hao-Chung Yang, Qifa Zhou, Lihong V. Wang

Joon-Mo Yang, Konstantin Maslov, Hao-Chung Yang, Qifa Zhou, Lihong V. Wang, "Endoscopic photoacoustic microscopy," Proc. SPIE 7177, Photons Plus Ultrasound: Imaging and Sensing 2009, $71770 \mathrm{~N}$ (24 February 2009); doi: 10.1117/12.810952

SPIE. Event: SPIE BiOS, 2009, San Jose, California, United States 


\title{
Endoscopic Photoacoustic Microscopy
}

\author{
Joon-Mo Yang ${ }^{1}$, Konstantin Maslov ${ }^{1}$, Hao-Chung Yang ${ }^{2}$, Qifa Zhou ${ }^{2}$ and Lihong V. Wang ${ }^{1 *}$ \\ ${ }^{1}$ Department of Biomedical Engineering, Optical Imaging Laboratory, Washington University in St. \\ Louis, One Brookings Drive, Campus Box 1097, St. Louis, Missouri, 63130-4899, USA
${ }^{2}$ Department of Biomedical Engineering, University of Southern California, 1042 Downey Way, University Park, DRB 130, Los Angeles, CA 90089-111, USA

\begin{abstract}
We present a concept and system implementation for endoscopic photoacoustic microscopy that enables minimally invasive diagnosis of internal organs. The system incorporates an in-house made single element ultrasonic transducer for photoacoustic signal detection and an optical fiber for light delivery into a tubular probe with a mechanical scanning unit. The implemented probe size for the distal end is $4.2 \mathrm{~mm}$ in diameter and $48 \mathrm{~mm}$ in length. We evaluated the system's performance by imaging a carbon fiber in clear and turbid media. We also demonstrated its ability to image actual biological tissues and its endoscopic applicability, by imaging abdominal surfaces and a large intestinal tract of a rat ex vivo. This study shows the system's potential for in vivo optical biopsy of tissue abnormalities, such as tumors, developed in internal organs.
\end{abstract}

Keywords: Endoscopic photoacoustic microscopy, minimally invasive, diagnosis, internal organs, tumors, optical biopsy, scanning mirror, micromotor.

\section{INTRODUCTION}

\subsection{Motivation}

Endoscopy is an important technique in practical medicine to diagnose internal organs, such as respiratory and gastrointestinal (GI) tracts. The most common technique is video endoscopy $y^{1,2}$ which can provide clear real time video images of organs' surface to operator. However, these video images lack depth information and are insufficient to diagnose diseased tissues that develop in endothelial tissues. An advanced technique to overcome this obstacle is endoscopic ultrasonography (EUS). An intravascular ultrasound (IVUS) probe $\mathrm{p}^{3-6}$ and gastrointestinal ultrasound probe $\mathrm{e}^{7}$, ${ }^{8}$ are representative tools for cardiovascular imaging and GI tract imaging, respectively. They can image very deep areas of organs, up to several centimeters, based on ultrasonographic techniques. However, this kind of ultrasound probe has some limitations for the early diagnosis of tissue's abnormalities, such as tumors, because its intrinsic contrast is based on the tissue's mechanical elastic properties. For this reason, novel optical endoscopic techniques have developed. Representative techniques are endoscopic optical coherence tomography (OCT) ${ }^{9-12}$ and confocal endoscopy ${ }^{13,14}$. These techniques can produce high resolution 3D images based on optical contrast, which is more sensitive than current techniques for detecting tissue's abnormalities. But their limitation is poor penetration depth because they utilize ballistic or quasi-ballistic photons. However, recently emerging photoacoustic endoscopy ${ }^{15-19}$ is expected to be much superior to other endoscopies because it is a hybrid imaging modality of optics and ultrasound. It combines the strong points of optical and ultrasound imaging so that it can image deep areas of organs with high resolution and optical absorption contrast.

\subsection{Review of previous studies}

* Corresponding author: lhwang@biomed.wustl.edu. ${ }^{1}$ These authors contributed equally to this work.

Photons Plus Ultrasound: Imaging and Sensing 2009, edited by Alexander A. Oraevsky, Lihong V. Wang, Proc. of SPIE Vol. 7177, 71770N · C 2009 SPIE · CCC code: 1605-7422/09/\$18 · doi: 10.1117/12.810952 
A photoacoustic endoscopic probe for $1 \mathrm{D}$ sensing was reported by Viator et.al ${ }^{17}$ in 2001 . Their system used a multimode optical fiber for light delivery and a PVDF film-based ultrasonic sensor for photoacoustic signal detection. A-line signals from optical phantoms were acquired with adequate SNR, but images could not be produced because the probe had no mechanical scanning mechanism. More recent studies ${ }^{18,19}$ reported photoacoustic and ultrasound pulseecho images of a rabbit's blood vessels ex vivo by using a commercial IVUS probe. The study showed photoacoustic endoscopy's potential to detect vulnerable plaque. However, it generated photoacoustic signals by illuminating the samples from outside (i.e., external light delivery); and also performed mechanical scanning by rotating the samples without a built-in mechanical scanning mechanism.

Incorporating illumination optics and a mechanical scanning mechanism is essential for practical application. However, no such method has yet been achieved, and its creation remains a challenge in this field.

\subsection{Technical challenges}

To realize a photoacoustic endoscope, the size restriction is the most challenging issue. Especially, its diameter should be less than $10 \mathrm{~mm}$ like other endoscope probes. Another challenge is to incorporate optical and ultrasonic elements into a single probe. Other endoscopic probes, such as endoscopic OCT or endoscopic ultrasonography, are in the pure optics or pure ultrasonic region, but photoacoustic endoscopy is in the hybrid domain of optics and ultrasound. Mechanical scanning is also important to produce an image. Achieving adequate SNR with a small aperture is another challenge because the external light impulse to generate the photoacoustic signal should be less than the ANSI safety limit $\left(20 \mathrm{~mJ} / \mathrm{cm}^{2} \text { per pulse }\right)^{20}$.

\section{MATERIALS AND METHOD}

\subsection{Concept of endoscopic photoacoustic microscopy}

In Figure 1 we present the concept of our endoscopic photoacoustic microscopy, which has a mechanical scanning mechanism with internal light delivery. The schematic shown is the system configuration of the probe's distal end, where an optical fiber for light delivery, an ultrasonic transducer for photoacoustic signal detection, and a geared micromotor for mechanical scanning are placed along the axis. Here, another key element is a scanning mirror, which performs a circumferential mechanical B-scan.

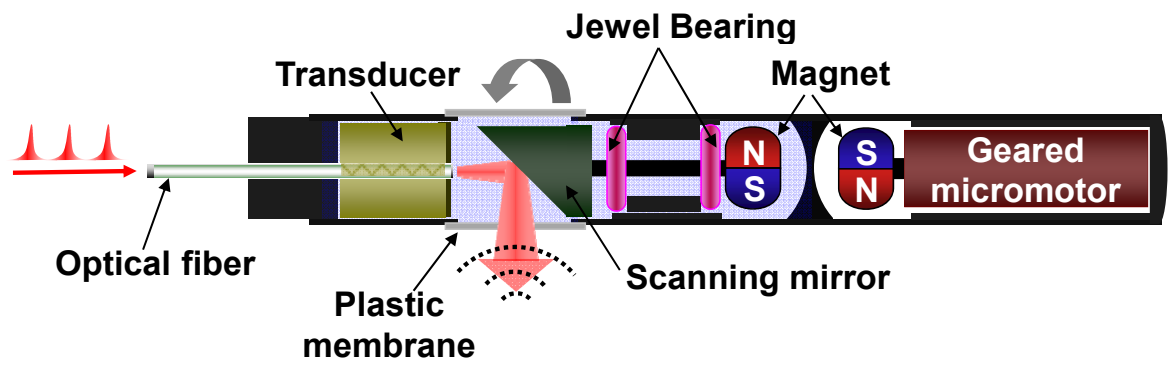

Figure 1: Schematic of the photoacoustic endoscope's distal end. The optical fiber passes through the central axis of the transducer's body. The scanning mirror and transducer's housing space is filled with deionized water but the geared micromotor is isolated from the water.

External laser pulses guided by a multimode optical fiber are emitted though the central hole of the ultrasonic transducer, reflected by the scanning mirror, and sent to the target tissue, finally generating photoacoustic waves. Some of these photoacoustic waves propagate to the scanning mirror, are reflected, and finally are detected by the ultrasonic transducer. So the scanning mirror reflects both laser pulses and ultrasonic waves during its constant rotational motion, and enables circumferential mechanical scanning. Here, it is important to fill the probe's inner space with a liquid medium to provide an ultrasonic matching medium for photoacoustic waves. To provide in-air working conditions for the micromotor, however its housing space is isolated by sealing it; but the torque required for the mirror's rotation is transferred from the micromotor through a magnetic coupling. 
This configuration enables mechanical scanning with static illumination optics and a static ultrasonic sensor, and does not require a flexible shaft-based external driving force. Most of all, the mechanical scanning concept employing the scanning mirror and coincident light illumination and ultrasonic detection scheme enables full B-scan with axial symmetry, which is the most typical scanning mechanism of existing endoscopes.

\subsection{The embodiment of a photoacoustic endoscope and its physical dimensions}

According to the aforementioned concept, we have constructed a photoacoustic endoscope; Figure 2-(a) shows a photo of it. A transducer module that includes an optical fiber [Figure 2-(b)], a scanning mirror [Figure 2-(c)], and a geared micromotor [Figure 2-(d)] were placed into a stainless steel tube with inner and outer diameters 3.25 and $3.76 \mathrm{~mm}$, respectively. The scanning mirror's housing space was filled with deionized water and sealed with a plastic membrane (50 $\mu \mathrm{m}$ thick, LDPE), which is suitable for both light and ultrasound transmission, and forms an imaging window. The probe shows its maximal thickness of $4.2 \mathrm{~mm}$ at the plastic imaging window, and the total length of the rigid part was $48 \mathrm{~mm}$. The endoscope's body, which is flexible, is formed by three bundles of cables, i.e., the optical fiber, the transducer's signal wires, and the micromotor's wires.

\section{(a)}

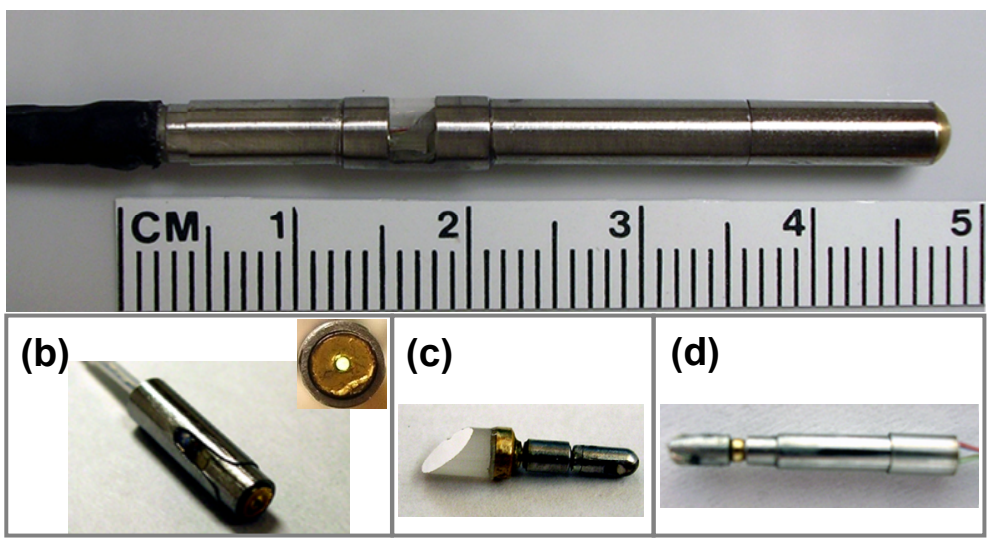

Figure 2: (a) Photo of the in-house made photoacoustic endoscopic probe (distal end). The rigid distal end size is $4.2 \mathrm{~mm}$ in diameter and $48 \mathrm{~mm}$ in length. (b) The optical fiber and ultrasonic transducer module. The inset is the front view of the transducer. (c) The scanning mirror. (d) The geared micromotor. (b) (d) are placed in the stainless steel tube of photo (a).

In the transducer module [Figure 2-(b)], an in-house made ultrasonic transducer $\left(\mathrm{LiNbO}_{3}, 40 \mathrm{MHz}\right.$ central frequency) that has a hole along its axis (see the inset of Figure 2-(b)) was utilized for photoacoustic signal detection; its outer diameter was $2.8 \mathrm{~mm}$ and it was $6.5 \mathrm{~mm}$ long. Its piezoelement's outer diameter was $2.0 \mathrm{~mm}$, and the central hole's diameter was $0.5 \mathrm{~mm}$. The photoacoustic signal from the transducer was sent to a low noise amplifier via a micro coaxial cable $(50 \Omega, 0.44 \mathrm{~mm}$ thick, Hitachi Cable Manchester). A multimode optical fiber with a core diameter of 365 um (0.22 NA) was utilized for light delivery and was placed along the transducer's central hole. The illumination optical fiber has no light focusing optics, such a lens, so emitted laser beam diverges with the half angle of $9.6^{\circ}$ and forms a spot diameter of $1.55 \mathrm{~mm}$ when it passes through the plastic imaging membrane.

In the scanning mirror module [Figure 2-(c)], an aluminum coated glass mirror (BK7) with an outer diameter of $3.0 \mathrm{~mm}$ was utilized and connected with a magnet through a metal shaft. The mirror's surface and material were good for both light and ultrasonic wave reflection, and its surface normal shows $45^{\circ}$ deflection to the endoscopic probe's axis. Considering the acoustic impedance (1.48 MRayl for water and 11.4 MRayl for BK7 glass) and longitudinal wave speeds $(1.50 \mathrm{~mm} / \mu \mathrm{s}$ for water, $5.10 \mathrm{~mm} / \mu \mathrm{s}$ for BK7 glass $)$ of the matching medium and the mirror material, the incidence angles of the acoustic rays propagated to the scanning mirror were in the range of total internal reflection (critical angle for longitudinal wave $=17.1^{\circ}$ ), so this configuration detects photoacoustic waves as efficiently as a direct facing detection mechanism. 
In the geared micromotor module [Figure 2-(d)], a DC micromotor with a geared box (gear ratio, 254:1; Namiki Precision, Inc.) was utilized; its outer diameter was $1.5 \mathrm{~mm}$ and it was $12.0 \mathrm{~mm}$ long. To provide magnetic coupling with the magnet described in the scanning mirror module [Figure 2-(c)], a similar magnet was attached at the tip of the motor's shaft.

For the tube's (housing) material, we chose stainless steel, which is not transparent to light, so there is some restriction in the field of view. Figure 3 presents a diagram of the probe's field of view, which is $250^{\circ}$ in angle and $3.0 \mathrm{~mm}$ in radius. The radial field of view was determined just by the data length ( 400 points) we set, rather than fundamental limitations of photoacoustic imaging, so it could be adjusted accordingly - the 400 data points correspond to $2.0 \mu \mathrm{s}$ in time because the sampling rate was set at $200 \mathrm{MHz}$. Considering acoustic speeds $(1.50 \mathrm{~mm} / \mu \mathrm{s}$ in water, $1.54 \mathrm{~mm} / \mu \mathrm{s}$ in soft biological tissue), it corresponds to $3.0 \mathrm{~mm}$ for water medium and $3.1 \mathrm{~mm}$ for soft biological tissues.

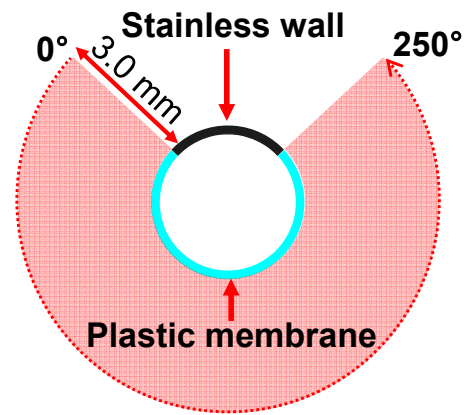

Figure 3: Field of view of the endoscopic probe; $3.0 \mathrm{~mm}$ in radius and $250^{\circ}$ in angle. The angular field of view was restricted by the stainless steel wall, and the optically and ultrasonically transparent plastic membrane forms an imaging window.

In Figure 4 we present a block diagram of the photoacoustic endoscopic probe and its peripheral systems. A dye laser pumped by an Nd:YLF laser is used as a pulsed light source, and the pump laser is triggered by the TTL signals from the micromotor's drive circuit. A cycle of the TTL signal that triggers the pump laser and a data acquisition card is generated by one rotation of the micromotor. Because $254 \mathrm{TTL}$ cycles are generated for one full rotation of the scanning mirror, which is determined by the geared micromotor's 254:1 gear ratio, a B-scan image is composed of 254 A-lines and is acquired by the high speed data acquisition card (Gage card) at a sampling rate of $200 \mathrm{MHz}$ (5 ns per data point) after signal amplification. For signal amplification, two serial low noise amplifiers with the total gains of $48 \mathrm{~dB}$ (ZFL$500 \mathrm{LN}+$, Mini-Circuits) were utilized. In this way, a set of B-scan images is acquired during the micromotor's constant rotational motion.

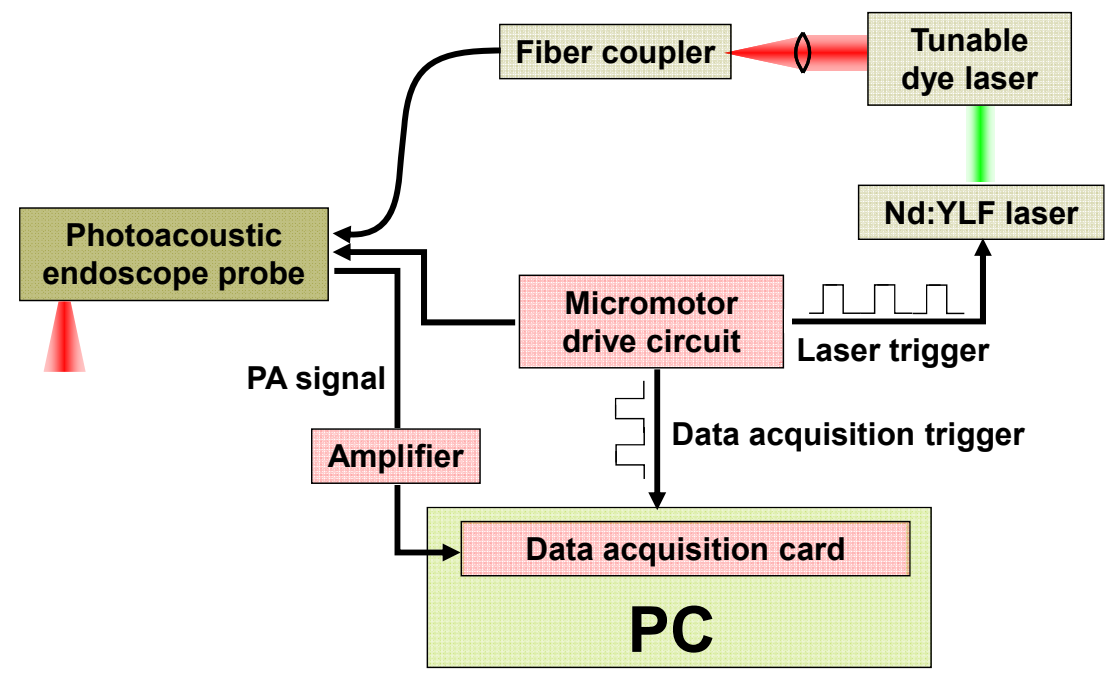

Figure 4: Schematic of the photoacoustic endoscopic probe and its peripheral systems. 


\subsection{Other experimental parameters and data processing method}

The dye laser's wavelength is tunable, but was set at $570 \mathrm{~nm}$, which is one of the isosbestic points in hemoglobin's absorption spectra, in this study. The laser beam's power was set in accordance with ANSI's safety limit $\left(20 \mathrm{~mJ} / \mathrm{cm}^{2}\right.$ per pulse) $)^{20}$, at $17.6 \mathrm{~mJ} / \mathrm{cm}^{2}$ at the sample's surface (i.e., plastic membrane). As explained before, a B-scan image is composed of 254 A-lines; however, because of the restricted field of view shown in Figure 3, 76 A-lines, which correspond to the stainless steel wall, were excluded when plotting the B-scan image. The angular step of two successive A-lines in a B-scan image is $1.42^{\circ}$, which was determined by the gear ratio (254:1). In this experiment, the scanning mirror's rotation speed was set at $2.6 \mathrm{~Hz}$.

To minimize noise, we applied a digital band pass filter $(10 \sim 70 \mathrm{MHz})$ to each A-line after data acquisition. The detected photoacoustic signals are bipolar, rather than unipolar, so we applied a Hilbert transform for each A-line to detect the envelope; and all B-scan images in this paper are plotted with the Hilbert transformed data.

\subsection{Carbon fiber imaging and ex vivo imaging of a rat}

To characterize the system's performance, we utilized a carbon fiber as a photoacoustic signal target. Its diameter was about $6 \mu \mathrm{m}$, so it can be regarded as a point target. As shown in Figure 5, we placed the endoscopic probe and the carbon fiber parallel in a liquid medium, and imaged the target at different positions (6 points) by moving it with a translational stage. We conducted the experiment in both clear (deionized water) and turbid (1\% intralipid solution; $\mu_{\mathrm{s}} \approx 14 \mathrm{~cm}^{-1}$ ) media, which is close to the reduced scattering coefficient of human's mucous tissue ${ }^{21}$.

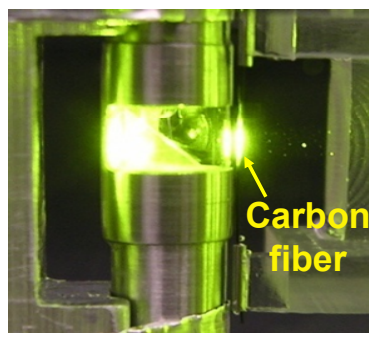

Figure 5: Photo showing the carbon fiber imaging experiment. The endoscope and carbon fiber are aligned parallel in the deionized water medium. The carbon fiber's position is horizontally movable by a translational stage.

To demonstrate the system's imaging capability for actual biological tissues, we imaged a rat's abdominal tissues in situ. First we sacrificed a rat (Sprague Dawley rats; 250 g; Harlan National Customer Service Center) by a chemical over dose (Pentobarbital, $120 \mathrm{mg} / \mathrm{kg}$, IP) and removed the hairs from its abdominal surface with a commercial hair removing lotion. To provide an acoustic matching medium for the probe, we spread acoustic gel between the endoscopic probe's imaging window and the animal's abdominal surface.

In addition, we imaged an excised portion of the rat's large intestinal tract to show the probe's endoscopic potential. After washing the sample with PBS, we inserted the probe into the inner tract of the excised large intestine and performed a B-scan.

All experimental animal procedures were carried out in compliance with the guidelines of the U.S. National Institute of Health. The laboratory animal protocol for this work was approved by the Animal Study Committee of Washington University in St. Louis.

\section{RESULTS AND DISCUSSION}

\subsection{System performance data}

In Figure 6 we present the results of the carbon fiber experiment. First, a typical A-line signal and its Hilbert transformed data for envelope detection are plotted in Figure 6-(a). With the Hilbert transformed data (red-dotted line), we determined the radial resolution of FWHM (-6 dB); and also determined SNR by analyzing peak intensity and noise fluctuation (standard deviation). In Figure 6-(b) and 6-(c), we present the carbon fiber's photoacoustic images in Cartesian/polar coordinate representation, respectively; and these images are plotted with the Hilbert transformed data. 
Figure 6-(d) is the transverse point spread function (PSF) of the carbon fiber, from which we determined the FWHM (-6 $\mathrm{dB}$ ) transverse resolution. In this way, we determined the system's SNR, radial resolution, and transverse resolution for the six carbon fiber's positions (the x-distances were measured from the surface of the plastic membrane); the results are plotted in Figure 6-(e) (g) with the averages of data for 30 B-scan images.

(a)

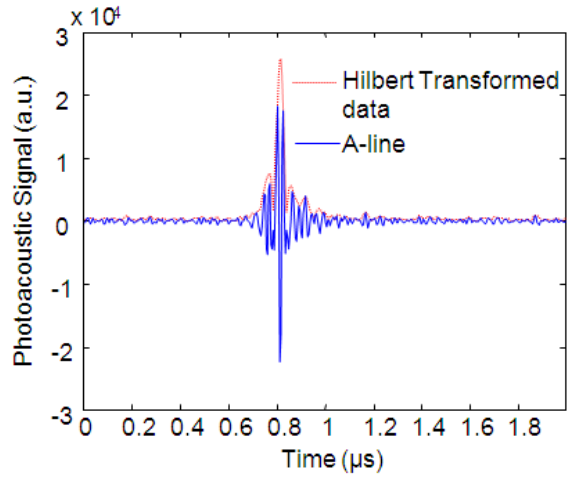

(d)

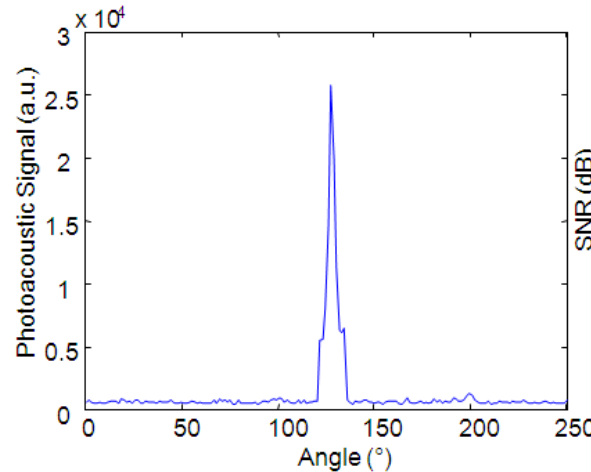

(b)

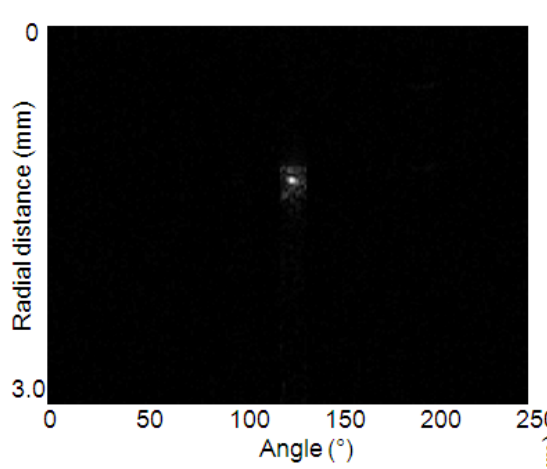

(e)

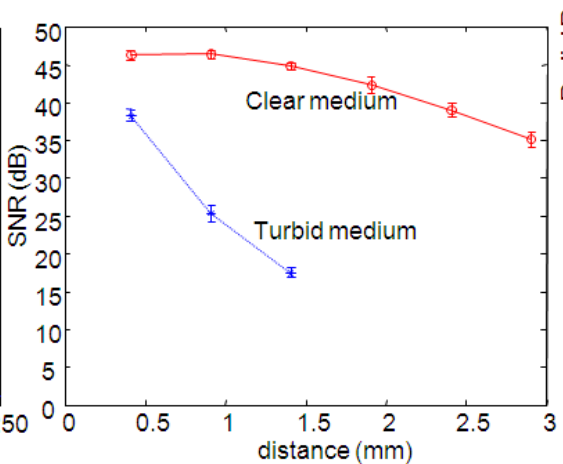

(c)

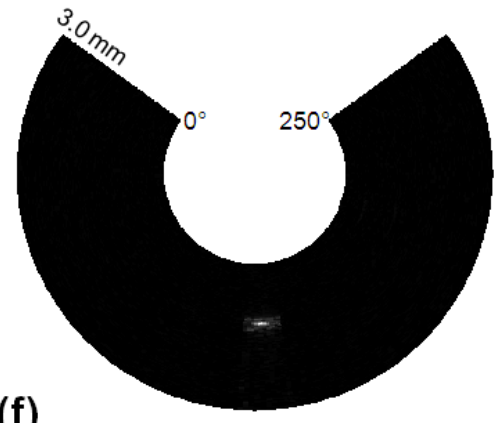

(f)

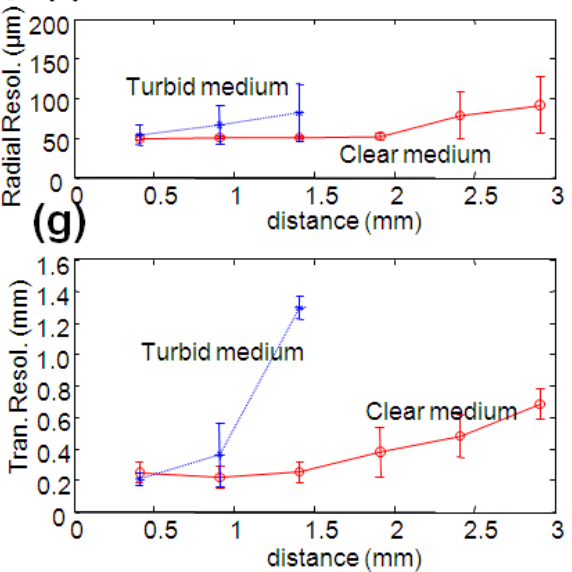

Figure 6: (a) Typical A-line (blue line) signal from the carbon fiber and its Hilbert-transformed data (red-dotted line). (b) Photoacoustic image of the carbon fiber: Cartesian coordinate representation. (c) Polar coordinate representation of the photo acoustic image (b). (d) Transverse PSF for the carbon fiber. (e) SNR vs. target distance for clear (deionized water) and turbid (1 \% intralipid; $\mu_{\mathrm{s}}^{\prime} \approx 14 \mathrm{~cm}^{-1}$ ) media. (f) Radial resolution vs. target distance. (g) Transverse resolution vs. target distance. The $\mathrm{x}$-distances were measured from the surface of the plastic membrane

In Figure 6-(e), the SNR for the clear medium starts at $46 \mathrm{~dB}$ near the plastic imaging window and decreases with distance to about $35 \mathrm{~dB}$. This is a natural result because of the diverging beam pattern from the optical fiber and the increment of the distance between the detector and the target. However, the SNR for the turbid medium starts at $38 \mathrm{~dB}$ and decreases quickly according to the distance. The turbid medium's poor SNR can be attributed to the low optical fluence delivered to the target. The data for the $4^{\text {th }} \sim 6^{\text {th }}$ points were undetectable, mainly because of the carbon fiber's fine size $(\sim 6 \mu \mathrm{m})$. Another reason is the quick decay of optical fluence in the turbid medium (optical fluence near the plastic imaging window was $\sim 17.6 \mathrm{~mJ} / \mathrm{cm}^{2}$ and, if the turbid medium in the tank is regarded as an infinite homogeneous turbid medium, it decays quickly according to the formula of $\exp \left(-\mu_{\mathrm{eff}} \mathrm{r}\right) / \mathrm{r}$ based on the diffusion theory. Here, $\mu_{\text {eff }}$ denotes the effective attenuation coefficient.).

In Figure 6-(f), we can see that the radial resolutions for the clear medium increase slowly over the target distance, ranging between $50 \mu \mathrm{m}$ and $90 \mu \mathrm{m}$. However, the radial resolution in the turbid medium becomes poor compared to that in the clear medium with an increasing the target distance, and ranges from $54 \mu \mathrm{m}$ to $80 \mu \mathrm{m}$ (the data for the $4^{\text {th }} \sim 6^{\text {th }}$ points are unavailable). 
Figure 6-(g) shows that the transverse resolutions in the clear medium increase gradually with an increasing target distance, but those in the turbid medium do more steeply. They range from $250 \mu \mathrm{m}$ to $700 \mu \mathrm{m}$ for the clear medium, and from $200 \mu \mathrm{m}$ to $1200 \mu \mathrm{m}$ for the turbid medium (the data for the $4^{\text {th }} \sim 6^{\text {th }}$ points are unavailable).

In photoacoustic imaging, the main factor that determines the spatial resolution is the system's acoustic parameters rather than its optical parameters. Because the acoustic properties of the deionized water and $1 \%$ intralipid medium are very close, we interpret that the poor resolution in the turbid medium arose from the poor SNR (i.e., poor optical fluence).

\subsection{In situ imaging results of the rat's abdominal tissues}

In Figure 7, we present in situ imaging results of the rat's abdomen. We imaged two areas of the abdomen, and present them in the upper and lower rows of the figure. The first column represents photographic images of the imaged area, and second and third columns are their photoacoustic images plotted in Cartesian and polar coordinate formats, respectively. In the photographic images, scanning direction and range are indicated as yellow-dotted arrows.

In Figure 7-(b), we can see many strong signal spots sourcing from blood vessels (BV1 BV3) at the shallow region of the abdominal surface, about $0.6 \mathrm{~mm}$ depths. In Figure 7-(e), in addition to the strong signals (BV1, BV2) near the surface (about $0.6 \mathrm{~mm}$ depths), a highly light-absorbing internal structure appears at a depth of about $1.8 \mathrm{~mm}$. These results show the deep imaging capability of our endoscopic probe with adequate SNR.

(a)

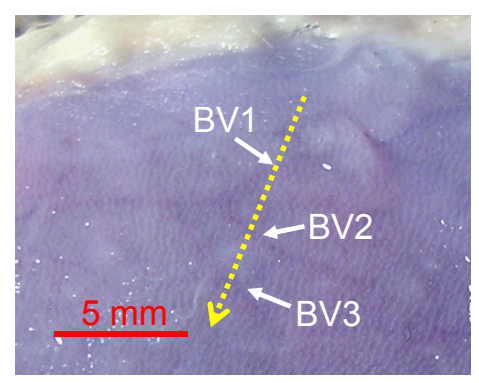

(d)

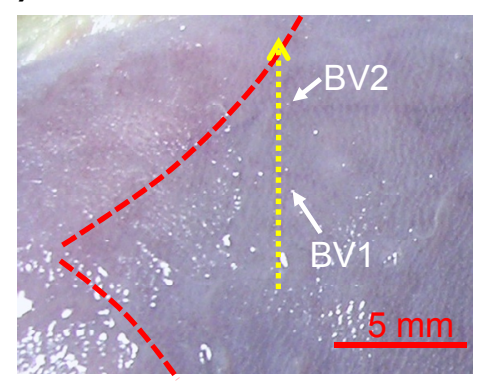

(b)

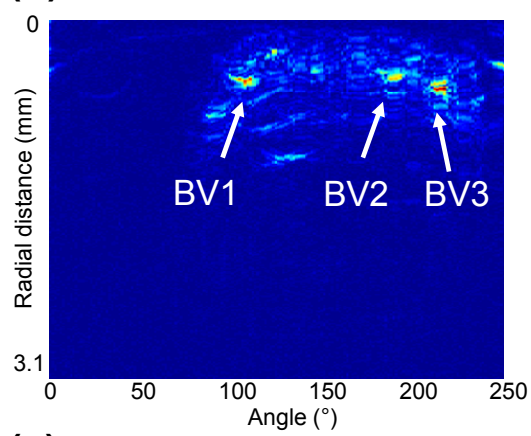

(e)

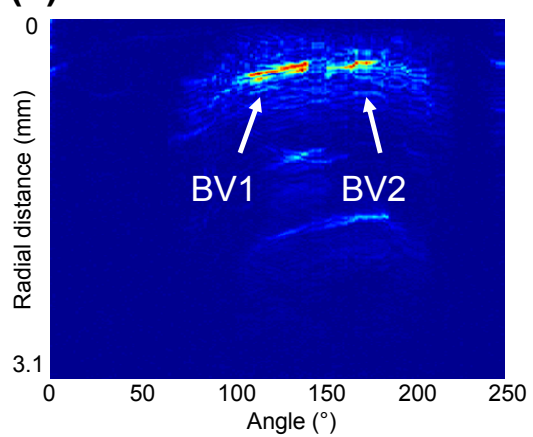

(c)

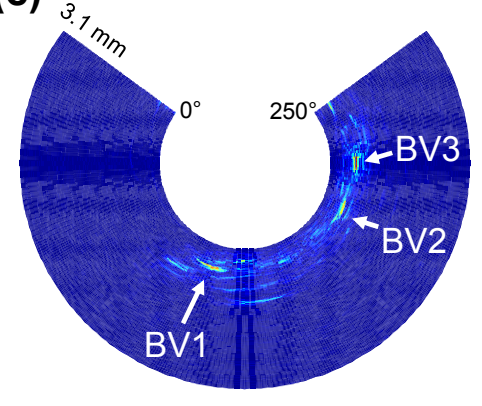

(f)

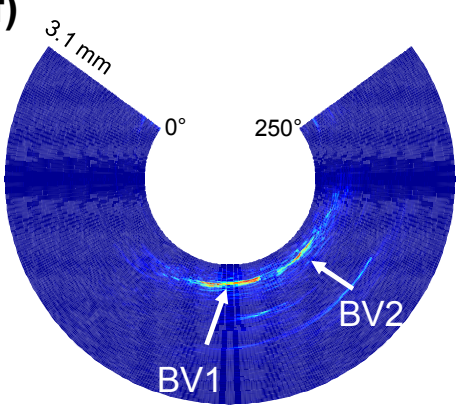

Figure 7: (a) Photo of imaging area 1. The yellow-dotted arrow shows the scanning direction and range. (b) Photoacoustic image of (a): Cartesian coordinate representation. (c) Polar coordinate representation of the photo acoustic image (b). (d) Photo of imaging area 2. The yellow-dotted arrow shows the scanning direction and range. The red-dotted line indicates the false ribs of the rat. (e) Photoacoustic image of (d): Cartesian coordinate representation. (f) Polar coordinate representation of the photo acoustic image (e). $\mathrm{BV}$, blood vessel.

\subsection{Ex vivo imaging result of the rat's large intestinal tract}

In Figure 8, we present ex vivo imaging result of the rat's large intestinal tract. Figure 8-(a) represents a photograph of the intact large intestinal tract, into which the endoscopic probe was inserted. We spread acoustic gel to improve better 
acoustic match and the scanning mirror's trajectory is indicated as a yellow-dotted arrow. In the photoacoustic image shown in Figure 8-(b), we can see the sample's morphology along the scanning line. Figure 8-(c) is a polar coordinate representation of the image (b). From this photoacoustic image, we estimate the intestinal wall's thickness at about 1.5 $\mathrm{mm}$.

(a)

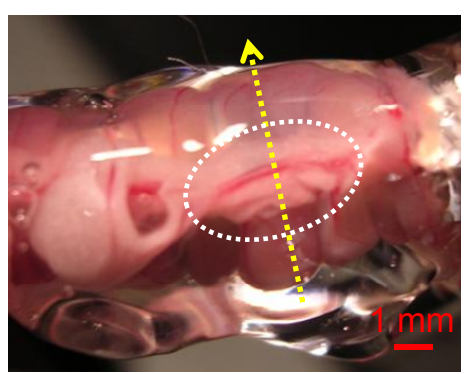

(b)

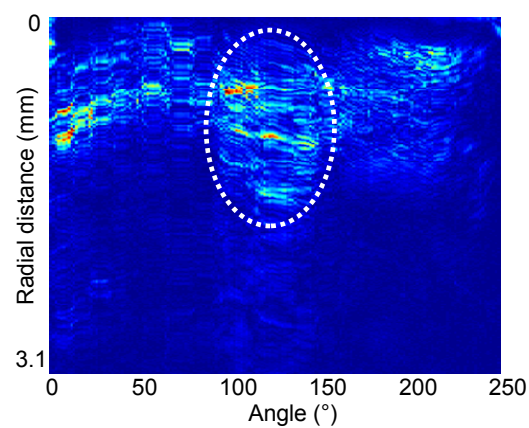

(c)

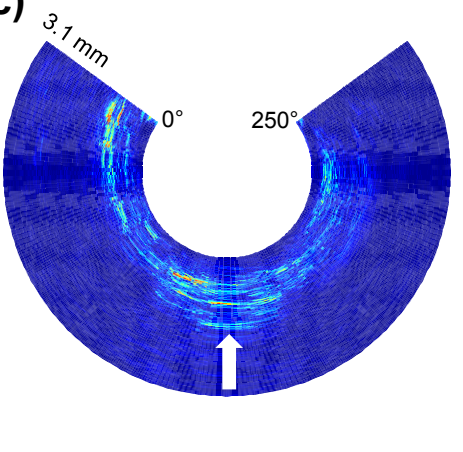

Figure 8: (a) Photo of the intact large intestinal tract into which the endoscope probe was inserted. The yellow-dotted arrow shows the scanning direction. (b) Photoacoustic image of (a): Cartesian coordinate. The white-dotted circle of (a) corresponds to that of (b). (c) Polar coordinate representation of the photo acoustic image (b). The area indicated by a white block arrow in (c) corresponds to the white -dotted circles in (a) and (b).

The ex vivo imaging results show our photoacoustic probe's endoscopic potential.

\section{CONCLUSIONS}

In summary, we have presented the concept and system implementation for endoscopic photoacoustic microscopy. The system used a mechanical scanning mechanism with internal light delivery, with a size of $4.2 \mathrm{~mm}$ in diameter and 48 $\mathrm{mm}$ in length. We characterized its imaging performance by imaging a carbon fiber, and achieved adequate SNR and spatial resolution. In this system's configuration, the key idea is to use a scanning mirror that reflects both lights and ultrasound waves and enables circumferential mechanical scanning. This study demonstrates the indirect signal detection concept that employs a scanning mirror, which is identical to the conventional scanning mechanism that detects signals by facing target tissues directly. Using the scanning mirror can make the system simple and stable for transmitting impulses and responses via the probe's body. The reliable mapping of the blood vessels in the in situ experiments implies the system's in vivo imaging capability. Most of all, the photoacoustic image of the rat's large intestinal tract shows the probe's endoscopic potential.

\section{ACKNOWLEDGEMENT}

This research was supported by the National Institutes of Health grants R01 NS46214 (BRP), R01 EB000712, R01 EB008085, and U54 CA136398 (Network for Translational Research). L.W. has a financial interest in Endra, Inc., which, however, did not support this work. The authors are grateful to Namiki Precision, Inc. for supporting the micromotor and Hitachi Cable Manchester for supporting the micro-coaxial cable.

\section{REFERENCES}

[1] M. Epstein, “Endoscopy: Developments in Optical Instrumentation,” Science 210 (4467), pp. 280-285 (1980).

[2] I. Belinsky, H. Shinya, and W. I. Wolff, "Colonofiberoscopy: Technique in Colon Examination," Am. J. Nurs. 73 (2), pp. 306-308 (1973).

[3] C. Strimike, "CE Credit: New Procedures: Understanding Intravascular Ultrasound," Am. J. Nurs. 96 (6), pp. 40-44 (1996). 
[4] S. J. Nicholls, I. Sipahi, P. Schoenhagen, T. Crowe, E. M. Tuzcu, and S. E. Nissen, "Application of intravascular ultrasound in anti-atherosclerotic drug development," Nat. Rev. Drug Discov. 5, pp. 485-492 (2006).

[5] A. Konig, M P. Margolis, R. Virmani, D. Holmes, and V. Klauss, "Technology Insight: in vivo coronary plaque classification by intravascular ultrasonography radiofrequency analysis," Nat. Clin. Pract. Card. 5, pp. 219-229 (2008).

[6] G. Sarno, A. Lerman, J.-H. Bae, C. Schukro, D. Glogar, P. M Margolis, M. Goethals, S. Verstreken, J. Bartunek, A. Koenig, W. Wijns, and M. Vanderheyden, "Multicenter assessment of coronary allograft vasculopathy by intravascular ultrasound-derived analysis of plaque composition," Nat. Clin. Pract. Card. 6, pp. 61-69 (2009).

[7] J. Menzel, W. Domschke, "Gastrointestinal Miniprobe Sonography: The Current Status," Am. J. Gastroeneterol. 95, pp. 605-616 (2000).

[8] A. Fritscher-Ravens, M. Mylonaki, A. Pantes, T. Topalidis, F. Thonke, and P. Swain, "Endoscopic UltrasoundGuided Biopsy for the Diagnosis of Focal Lesions of the Spleen,” Am. J. Gastroeneterol. 97, pp. 2768-2775 (2002).

[9] G. J. Tearney, S. A. Boppart, B. E. Bouma, M. E. Brezinski, N. J. Weissman, J. F. Southern, and J. G. Fujimoto, "Scanning single-mode fiber optic catheter-endoscope for optical coherence tomography," Opt. Lett. 21, pp. 543-545 (1996).

[10] G. J. Tearney, M. E. Brezinski, B. E. Bouma, S. A. Boppart, C. Pitris, J. F. Southern, and J. G. Fujimoto, "In vivo endoscopic optical biopsy with optical coherence tomography," Science 276, pp. 2037-2039 (1997).

[11] W. Jung, D. T. McCormick, Y.-C. Ahn, A. Sepehr, M. Brenner, B. Wong, N. C. Tien, and Z. Chen, "In vivo threedimensional spectral domain endoscopic optical coherence tomography using a microelectromechanical system mirror," Opt. Lett. 32 (22), pp. 3239-3241 (2007).

[12] S. H. Yun, G. J. Tearney, B. J. Vakoc, M. Shishkov, W. Y. Oh, A. Desjardins, R. Chan, M. Suter, J. Evans, I. K. Jang, N. S. Nishioka, J. F. de Boer, and B. E. Bouma, "Comprehensive volumetric optical microscopy in vivo," Nat. Med. 12, pp. 1429-1433 (2006).

[13] R. Kiesslich, M. Goetz, M. Vieth, P. R. Galle, and M. F. Neurath, "Technology Insight: confocal laser endoscopy for in vivo diagnosis of colorectal cancer," Nat. Clin. Pract. Oncol. 4, pp. 480-490 (2007).

[14] J. T. C. Liu, M. J. Mandella, H. Ra, L. K. Wong, O. Solgaard, G. S. Kino, W. Piyawattanametha, C. H. Contag, and T. D. Wang, "Miniature near-infrared dual-axes confocal microscope utilizing a two-dimensional microelectromechanical systems scanner," Opt. Lett. 32 (3), pp. 256-258 (2007).

[15] H. F. Zhang, K. Maslov, G. Stoica, and L. V. Wang, "Functional photoacoustic microscopy for high-resolution and noninvasive in vivo imaging," Nat. Biotech. 24, pp. 848-851 (2006).

[16] L. V. Wang, "Prospects of photoacoustic tomography," Med. Phys. 35 (12), pp. 5758-5767 (2008).

[17] J. A. Viator, G. Paltauf, S. L. Jacques, and S. A. Prahl, "Design and testing of an endoscopic photoacoustic probe for determination of treatment depth after photodynamic therapy," Proc. SPIE 4256, pp. 16-27 (2001).

[18] S. Sethuraman, S. R. Aglyamov, J. H. Amirian, R. W. Smalling, and S. Y. Emelianov, "Intravascular photoacoustic imaging using an IVUS imaging catheter," IEEE Tran. Ultra. Ferro. Freq. Contr. 54 (5), pp. 978-986 (2007).

[19] S. Sethuraman, J. H. Amirian, S. H. Litovsky, R. W. Smalling, and S. Y. Emelianov, "Spectroscopic intravascular photoacoustic imaging to differentiate atherosclerotic plaques," Opt. Exp. 16 (5), pp. 3362-3367 (2008).

[20] American National Standards Institute, "American National Standard for the Safe Use of Lasers," ANSI Standard Z136.1, New York (2000).

[21] A. N. Bashkatov, E. A. Genina, V. I. Kochubey, and V. V. Tuchin, "Optical properties of human skin, subcutaneous and mucous tissues in the wavelength range from 400 to $2000 \mathrm{~nm}$," J. Phys. D: Appl. Phys. 38 (15), pp. 2543-2555 (2005). 